\title{
Multi-homing in Heterogeneous Wireless Access Networks: A Stackelberg Game for Pricing
}

\author{
Joohyung Lee \\ Department of Software, Gachon University \\ Seongnam 13120, South Korea \\ [e-mail: j17.lee@gachon.ac.kr] \\ *Corresponding author: Joohyung Lee
}

Received August 3, 2017; revised September 28, 2017; revised November 2, 2017; accepted November 15, 2017; Published May 31, 2018

\begin{abstract}
Multimedia applications over wireless networks have been evolving to augmented reality or virtual reality services. However, a rich data size compared to conventional multimedia services causes bandwidth bottlenecks over wireless networks, which is one of the main reasons why those applications are not used widely. To overcome this limitation, bandwidth aggregation techniques, which exploit a multi-path transmission, have been considered to maximize link utilization. Currently, most of the conventional researches have been focusing on the user end problems to improve the quality of service (QoS) through optimal load distribution. In this paper, we address the joint pricing and load distribution problem for multi-homing in heterogeneous wireless access networks (ANs), considering the interests of both the users and the service providers. Specifically, we consider profit from resource allocation and cost of power consumption expenditure for operation as an utility of each service provider. Here, users decide how much to request the resource and how to split the resource over heterogeneous wireless ANs to minimize their cost while supporting the required QoS. Then, service providers compete with each other by setting the price to maximize their utilities over user reactions. We study the behaviors of users and service providers by analyzing their hierarchical decision-making process as a multileader-, multifollower Stackelberg game. We show that both the user and service provider strategies are closed form solutions. Finally, we discuss how the proposed scheme is well converged to equilibrium points.
\end{abstract}

Keywords: Multi-homing, heterogeneous access networks, joint pricing and load distribution, resource allocation, power consumption, Stackelberg game

An earlier version of this paper was presented at the IEEE Wireless Communications and Networking Conference (WCNC): - Track 3: Mobile and Wireless Networks, 2015. This work was supported in part by the Korean MSIT (Ministry of Science and ICT), under the National Program for Excellence in SW (2015-0-00932), supervised by the IITP (Institute for Information \& communications Technology Promotion) and in part by the Basic Science Research Program of the National Research Foundation of South Korea under Grant NRF-2018R1C1B6001849.I 


\section{Introduction}

In recent times, the demand for rich multimedia applications such as augmented reality (AR) and virtual reality (VR) have been growing increasingly with innovations in mobile terminals and wireless communication technologies [1-3]. Nevertheless, to support such rich multimedia applications over wireless networks (ANs), mobile users often suffer from a problem of quality of service (QoS) because of bandwidth bottlenecks [1]. To alleviate this bandwidth bottlenecks from the limited wireless resources, a multi-homing technique, which enables mobile users to aggregate heterogeneous wireless resources, has been steadily gaining importance in the market owing to its various benefits such as load balancing and bandwidth aggregation [4, 5]. Subsequently, there are several ways to implement the multi-homing techniques, which are as follows: multipath transmission control protocol (TCP) [1, 2], streaming control transmission protocol (SCTP) [1, 5, 6, 7], and quick user datagram protocol Internet connections (QUIC) [1, 8]. Hence, in an area overlapped by heterogeneous wireless ANs, a mobile terminal with multiple interfaces can obtain its required bandwidth for rich multimedia applications such as AR and VR streaming from heterogeneous wireless ANs using its multi-homing capability.

Various forms of resource allocation problems have been studied in literature while trying to attain optimal operation of the multi-homing techniques taking into account various parameters including wireless channel conditions, available bandwidth, power efficiency, and price. Specically, several game-theoretic approaches have been proposed in recent times for analyzing resource competition between multiple mobile users with multi-homing capability [9-12]. The works of López and Agüero et al. [9] modeled a resource allocation game for achieving load balancing of heterogeneous wireless networks with the consideration of link capacity and the number of users. In [10], a game-theoretic approach is applied to a device to device (D2D) environment for bandwidth aggregation in the multi-homing technique where a competition among users is considered. The Nash bargaining game for reducing power consumption of heterogeneous wireless ANs among service providers was proposed in [11]. In recent times, by taking into account the interest of mobile users and service providers at the same time, a resource competition game for the multi-homing technique was proposed as our preliminary work [12], which is the closest to this paper. In this work, a joint pricing and load distribution problem of multi-homing was studied to maximize individual interests. Here, since they assumed that there is a single service provider, pricing competition among multiple service providers was not considered. Moreover, it proposed a greedy algorithm based on an exhaustive search, which returns sub-optimum strategies. Specifically, due to the discontinuity and no monotonic nature of optimization problem at leader level, the sub-optimum solution can be obtained by an iterative search algorithm where the proposed algorithm is started with selecting grid points of pricing strategy set with arbitrary grid size. Therefore, this work does not provide optimal solution and the rigorous mathematical proof of existence and uniqueness of equilibrium points. The quality of solution in this work is highly impacted by the grid size for iterative search. Thus, there was further room to improve the work in [12], which inspired our work.

In this study, we propose a novel pricing and load distribution mechanism for multi-homing in heterogeneous wireless ANs, considering user's rate allocation, user delay, and price for rate allocation as well as service provider revenue and power consumption cost for rate allocation. To solve the problems in the conventional works, the proposed scheme considers users with 
multi-interfaced terminals and multiple service providers, which own different wireless ANs. Through a multileader and multifollower Stackelberg game, users cooperatively decide how

Table 1. Notation for Formulation

\begin{tabular}{|c|c|}
\hline Notations & Explanation \\
\hline \hline $\mathrm{I}$ & Set of service providers \\
\hline $\mathrm{J}$ & Set of heterogeneous access networks \\
\hline $\mathrm{r}$ & Milocated rate vector over the access networks of service providers \\
\hline$r^{\min }$ & Minimum bandwidth requirement for supporting all users \\
\hline $\mathrm{P}$ & $\begin{array}{c}\text { Pricing vector per bandwidth at interface mapped into access networks of service } \\
\text { providers }\end{array}$ \\
\hline$P_{\text {max }, i, j}$ & Maximum power consumption of BS $j$ of service provider $\mathrm{i}$ \\
\hline$C_{i, j}$ & Available bit rate of service provider $i$ over access network $j$ \\
\hline
\end{tabular}

much amount of bandwidth should be aggregated from all available wireless ANs within the service area to maximize the proposed utility, taking into consideration the price of the resource allocation and the delay penalty at the required bandwidth. Further, multiple service providers compete with each other by setting the optimal price of resource allocation to maximize their utility, considering the profit and cost of power consumption response to the user reaction. In accordance, for service providers the proposed price decision mechanism can select the optimal price for resource allocation in multi-homing, considering their revenue and cost as well as guaranteeing user minimum QoS. Compared to the work in [12], since this paper considers multiple service providers in competitive situation, through rigorous game-theoretic analysis, we provide closed form solutions of optimal strategies of mobile users and service providers and prove that the proposed approach converges to a unique equilibrium solution. The numerical results represent existence and uniqueness of the NE of the pricing game among service providers. Further, the behavior of optimal strategies according to the number of service providers is depicted. Finally, we show that optimal load distribution and pricing based on a well-defined utility function can maximize the payoff for all participating mobile users and service providers at the equilibrium of the game.

The remainder of this paper is organized as follows. We describe the detailed system model and design utilities of users and service providers in Section 2. In Section 3, we briefly explain the Stackelberg game based on the proposed utilities. In Section 4, the joint pricing and load distribution problem is formulated as a multileader and multifollower Stackelberg game, including theorems, propositions, and some algorithms to obtain the user and service provider optimal strategies. In Sections 5 and 6, we present the theoretical analysis of the proposed Stackelberg game, prove the existence and uniqueness of the equilibrium solution, and present numerical results under two different wireless ANs, respectively. Finally, Section 7 concludes the paper.

\section{System Model}

In this section, we address the problem of rate allocation over multiple ANs from several alternative perspectives. Specific notations for formulation are described in Table $\mathbf{1}$. We consider a set of service providers $I=\{1,2, \ldots, I\}$ that own various heterogeneous ANs $J=\{1$, $2, \ldots, J\}$, which represents several wireless technologies (for example, WLAN, 3G, and LTE). We call this the interface. The available bit rate of each AN can be expressed in the matrix 
form: $\mathrm{C}=\left\{C_{i, j}\right\}_{\mathrm{I} \times \mathrm{J}}$, where each element $C_{i, j}$ is the available bit rate of service provider $i$ over the AN $j$. Here, a service provider can provide the available bandwidth to users who are willing to use the bandwidth with the given price $p_{i, j}$. In this case, a service provider broadcasts the

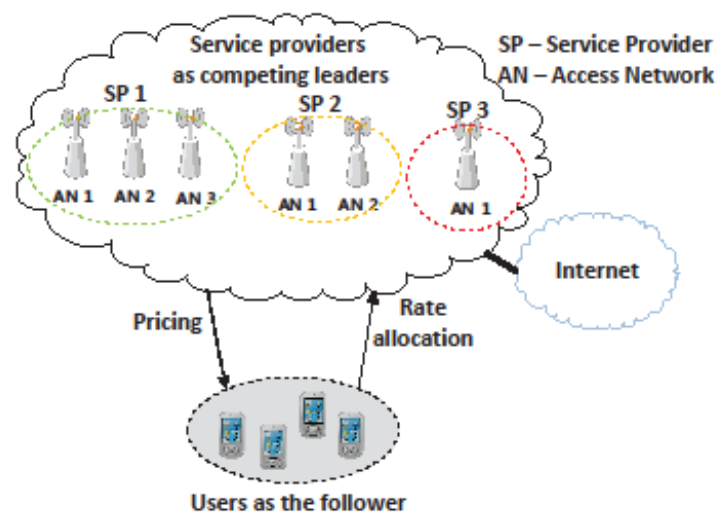

Fig. 1. Network architecture for a Stackelberg game for pricing and load distribution in multi-homing

availability of the bandwidth with the price to be paid over a dedicated control channel. Basically, in wireless networks, the users, who are in different locations, might experience different channel condition. Here, we consider that the users are willing to enjoy group-rich multimedia services in the same geographical area. For instance, such scenario can include that the users are in a class room and about to receive same VR educational contents in that area. In addition, users are in a meeting room to receive VR conference services. In this scenario, since we only focus on the population of a certain small area, we simplify our model to consider the users in a group experiencing the same channel condition by ignoring minor fading effect [13]. This model can be extended to cover multiple groups with different geographical areas and different channel condition as our future work. Thus, the users can access all ANs in I of service providers in J. In addition, in the proposed scenario, users are receiving same contents and sharing a common goal. For instance, the users in a class room aim to learn VR educational contents efficiently. Similarly, the users in a meeting room aim to conduct VR conference successfully. Thus, such scenario provides an incentive to the users to cooperative each other for their common goal. Accordingly, we assume that all the users request the same amount of bandwidth for service and are willing to cooperate with each other for maximizing social welfare so that their optimal rate vector over ANs would be the same ratio. Here, the requested minimum rate requirement for supporting the requested service over all users is denoted by $r^{\min }$. Besides, total aggregated rate over all ANs is $\sum_{i \in \mathrm{I}} \sum_{j \in \mathrm{J}} r_{i, j}$, and by (12) such aggregated rate is set to $r^{\mathrm{min}}$ where $r_{i, j}$ is the allocated rate vector over AN $j$ of the service provider $i$. That is the total aggregated rate over all ANs should meet the $r^{\text {min }}$ for guaranteeing the QoS.

Fig. 1 illustrates the network architecture for providing service to the users by multi-homing. A data flow for the provided service should be partitioned by the rate allocation to each heterogeneous AN. Multi-homed devices can then aggregate the partitioned data flow through multiple interfaces.

\subsection{Net Utility Function of Users (Social Welfare)}

By utilizing resources of simultaneously available multiple ANs through bandwidth aggregation, users can support an emerging application such as VR and AR, which basically require more bandwidth as well as lower latency compared to conventional video streaming. 
This bandwidth aggregation in the proposed work can be enabled by designing a striping mechanism in transport layer (i.e., multi-path TCP). It should be noted that the proposed works is not confined to specific striping mechanism. Based on this assumption, users aim at maximizing the social utility, which is coupled with the delay cost, price to pay, and battery consumption, while ensuring QoS. From the perspective of users, the total social cost for services needs to be arrived at using delay penalty as well as the price to pay for services. First, we consider the cost of delay such as latency.

In the delay model, we consider a general delay penalty model from [14] as below:

$$
D_{i, j}\left(r_{i, j}\right)=\frac{1}{C_{i, j}}\left(r_{i, j}-C_{i, j}\right),
$$

where $r_{i, j} \in\left[0, C_{i, j}\right]$. Therefore, the proposed utility function which represents the net utility or social welfare of the users is presented as below:

$$
\begin{aligned}
U_{\text {user }}(r)=\xi \log \left(1+\sum_{i \in \mathcal{I}} \sum_{j \in \mathcal{J}_{i}} r_{i, j}\right)-\kappa \sum_{i \in \mathcal{I}} \sum_{j \in \mathcal{J}_{i}} D_{i, j}\left(r_{i, j}\right) \cdot r_{i, j} \\
-\sum_{i \in \mathcal{I}} \sum_{j \in \mathcal{J}_{i}} p_{i, j} \cdot r_{i, j},
\end{aligned}
$$

where $\xi$ and $\kappa$ are the weight factors ( $\geq 0$ ) of the satisfaction from the bandwidth and delay, respectively.

There are two major motivations for using this utility function. First, since the function is a concave function from Proposition 2.1, it guarantees existence of a global optimal point. Second, the adjustable parameters $\xi$ and $\kappa$ provide flexibility to fit the function with different priorities for satisfaction from the bandwidth and delay. Moreover, in bandwidth aggregation, re-ordering problem is very important issue. This re- ordering risk can be happened when there is load imbalance between multi-path. In the proposed work, we can control the delay impact by adjusting parameter $\kappa$. That is, difference of delay in multipath can be managed to alleviate impact of re-ordering problem.

Proposition 1 The utility function $U_{\text {user }}$ of users is concave in $r$.

Proof

To prove the concavity of a multi-variable function, we have to check the negative semi-definiteness of Hessian matrix [15]. If Hessian of $U_{\text {user }}(r)$ is negative semidefinite for all $r \in R$, then $U_{\text {user }}(r)$ is concave. The Hessian of $U_{\text {user }}(r)$ is expressed as $H\left(U_{\text {user }}(r)\right)=\left\{t\left({ }_{i, j}\right),(l, m)\right\}$, $0 \leq i, l \leq \mathrm{I}, 0 \leq \mathrm{i} \leq \mathrm{J}_{i}$ and, $0 \leq m \leq \mathrm{J}_{i}$ where

$$
t_{(i, j),(l, m)}=\frac{\partial^{2} U_{u s e r}(r)}{\partial r_{i, j} \partial r_{l, m}}=\left\{\begin{aligned}
\frac{-2 \kappa}{C_{i, j}} & \text { if }(i, j)=(l, m) \\
0 & \text { if }(i, j) \neq(l, m) .
\end{aligned}\right.
$$

The Hessian matrix $H\left(U_{\text {user }}(r)\right)$ is a diagonal matrix in which only the main diagonal entries $\left.t\left({ }_{i, j}\right),{ }_{l, m}\right)$ are always negative. Therefore $z^{T} H\left(U_{\text {user }}(r)\right)<0$ for all non-zero vectors $z$. Consequently, we prove the concavity of the utility function $U_{\text {user }}$ in $r$.

\subsection{Utility Function of Service Providers}

We introduce a new general utility function of service providers by considering energy efficiency. Here, a base station (BS) consists of two types of power consumptions-fixed power consumption and adaptive power consumption-that are proportional to BS utilization [16]. Let 
the $P_{\max , i, j}$ be the maximum operational power of BS $j$ of service provider $i$ when it is fully utilized. And, the portion of fixed power consumption to $P_{\text {max }, i, j}$ is defined as $q_{i, j}$. Then, total power consumption of a BS is given as follows:

$$
P_{B S, i, j}(x)=\left(1-q_{i, j}\right) \cdot x / C_{i, j} \cdot P_{\max , i, j}+q_{i, j} \cdot P_{\max , i, j},
$$

where $q_{i, j} \in[0,1]$ and $x$ is the allocated rate to a BS.

Here, $P_{\text {max,i,j }}$ includes power consumptions for transmit antennas as well as power ampliers, cooling equipment and so on. Each AN has a common property that there are different $P_{\max , i, j}$, $C_{i . j}$, and $q_{i, j}$.

In the sense of service provider, the total utility function needs to be modeled by the cost of power consumption as well as the total revenue from the subscribers. Therefore, a novel utility function of a service provider is presented as shown below:

$$
U_{\text {ser }, i}=\sum_{j \in \mathcal{J}} p_{i, j} \cdot r_{i, j}-\sum_{j \in \mathcal{J}} c_{p} \cdot P_{B S, i, j}\left(r_{i, j}\right),
$$

where $c_{p}$ is the cost of the power consumption, and we assume that $c_{p}$ is a constant positive value and is provided by an electricity retailer.

\section{A Game-theoretic Approach: Stackelberg Game}

In this section, we introduce a game-theoretic approach to analyze our system model. Specifically, we describe the basic knowledge about the Stackelberg game to formulate the joint pricing and load distribution problem specified in sections 4 and 5 .

\subsection{Stackelberg Game Formulation}

In a proposed Stackelberg game, there are multiple leaders who hold strong positions and can impose their own strategies. Then, there is another player called as the follower, who reacts to the leader strategies. In our system model, a service provider takes the role of the leader. The service provider makes its own pricing strategy p to impose cost on the users who use AN interfaces for a service. In this case, users as followers decide the rate allocation for each interface based on the leader strategies to maximize their social welfare by cooperation, deciding on which service providers to procure bandwidth from and how much bandwidth to procure. Therefore, for the given pricing strategies $\mathbf{p}=\left\{p_{i, j}\right\}_{I \times \mathrm{J}}$ of service providers, we define the best response function $B_{f o l}$ of followers as:

$$
r^{*}=B_{\text {fol }}(p)=\arg \max _{\mathbf{r} \in \mathcal{R}} U_{\text {user }},
$$

where $\mathcal{R}$ denotes a set of possible rate allocation strategies.

From the leaders' point of view, the leader (a service provider) $i$ wants to maximize its utility by choosing a proper strategy. Therefore, the optimal pricing strategy $p^{*}{ }_{i}$ could be expressed as:

$$
p_{i}^{*}=\arg \max _{\mathbf{p}} U_{\text {ser }, i}\left(p_{i}\right)
$$

where $p_{i}=\left\{p_{i, 1}, p_{i, 2}, \ldots, p_{i, J}\right\}$. However, the utility function of the leader $U_{\text {lead }}$ not only depends on its own strategy, but also depends on the allocated rate by followers determined at (6). Then, equation (7) could be rewritten more specifically,

$$
p_{i}^{*}=\arg \max _{\mathbf{p}} U_{\text {ser }, i}\left(p_{i}, r^{*}(\mathbf{p})\right) \text {. }
$$

It means that the leader needs to consider the best response of the followers for the pricing strategy imposed to decide the optimal strategy. Furthermore, we also consider that multiple leaders compete with each other by setting their own price. Therefore, other service providers 
choose another criterion for deciding their strategies. Here, to find optimal strategies of service providers, they need to know the complete pricing strategies of the all other service providers. In the real world, it is not easy to obtain this information from competitors. One approach to handle this issue, we adopt a centralized broker concept, which gathers status information (e.g., $P_{B S, i, j}, C_{p}$ ) from all service providers and open such information to the all players. Accordingly, via the centralized broker, all this status information can be opened to all players in the game. The goal of this game is to find the Stackelberg equilibrium (SE) point, which is in pure strategies of all participating players. At SE point(s), both leaders and followers have no reason for deviating. For future work, by addressing practical scenario, this model can be extended to cover heterogeneous users with different channel conditions (i.e., large scale fading and small scale fading etc.,) due to their mobility. In addition, the proposed work is categorized as a static game model where service providers move one time simultaneously. Thus, the game with sequential move of service providers can be considered as dynamic games.

\subsection{Stackelberg Equilibrium}

For the formulated Stackelberg game, a pair of leader and follower strategies $\left(p^{S E}\left(=p_{1}{ }^{S E}, \ldots p_{i}{ }^{S E}\right)\right)$ is called SE if

$$
p_{i}^{S E}=\arg \max _{\mathbf{p}} U_{\text {ser }, i}\left(p_{i}, B_{\text {fol }}(\mathbf{p})\right),
$$

and $r^{S E}=B_{f o l}\left(p^{S E}\right)$. To find the SE, first, we solve the best response function (6) to find the relationship between the leader and the follower strategies. Based on that relationship, we can obtain the optimal pricing strategy $p_{i}^{*}$ by solving (8) and finding the SE points.

\section{Problem Formulation}

In this section, we use backward induction to analyze the performance of the Stackelberg game based on the utility functions defined in the previous section. Given that $\mathbf{p}$ is decided by service providers (multiple leaders) as well as other weight factors, users (multiple followers) maximize their own utility $\left(U_{\text {user }}\right)$ by selecting their rate allocation vectors while guaranteeing a minimum QoS requirement.

From the utility function of users defined at (2) with the given p, the best response of followers in (6) could be rewritten as:

$$
\mathbf{r}=\arg \max _{\mathbf{r} \in \mathcal{R}} U_{\text {user }}(\mathbf{r}, \mathbf{p}),
$$

where $\mathbf{p}$ is the given pricing strategies of service providers, $\mathcal{R}$ is an open set:

$$
\mathcal{R}=\left\{r \in \mathbb{R}^{I, J} \mid r_{i, j} \geq 0, r_{i, j} \leq C_{i, j}, \sum_{i \in I} \sum_{j \in J} r_{i, j}=r^{\min }, \forall i \in I, \forall j \in J\right\} .
$$

To find the best response rate allocation for the given price, we will transform (10) into convex optimization problem [15].

\subsection{Optimization Problem at the Follower-Level}

Since a user utility function $U_{\text {user }}$ is a concave function, solving the best response function of users in (10) could be considered as a convex optimization problem as follows: 


$$
\begin{aligned}
\max _{\mathbf{r}} & U_{\text {user }}(\mathbf{r}, \mathbf{p}) \\
\text { s.t. } \sum_{i \in I} \sum_{j \in J} r_{i, j} & =r^{\min }, \text { for } \forall i \in I, \forall j \in J, \\
r_{i, j} \geq 0, & \text { for } \forall i \in I, \forall j \in J, \\
r_{i, j} \leq C_{i, j}, & \text { for } \forall i \in I, \forall j \in J .
\end{aligned}
$$

The objective function (11) is concave and the problem has linear constraints. Therefore, the transformed problem as $\min _{\mathbf{r} \in \mathcal{R}}-U_{\text {user }}(\mathbf{r}, \mathbf{p})$ is a convex optimization problem, which creates a local and a global maximum as well. Hence, the transformed problem is the convex optimization problem. The basic idea in Lagrange duality [15] is to relax the original problem (11)-(14) by transferring the constraints to the objective in the form of a weighted sum. We then define a Lagrangian associated with the above problem to be

$$
\begin{aligned}
L(\mathbf{r}, \lambda, \sigma, \mu)= & U_{\text {user }}(\mathbf{r}, \mathbf{p})+\sum_{i \in I} \sum_{j \in J} \lambda_{i, j}\left(r_{i, j}-r^{\mathrm{min}}\right) \\
& +\sum_{i \in I} \sum_{j \in J} \sigma_{i, j} r_{i, j}+\sum_{i \in I} \sum_{j \in J} \mu_{i, j}\left(C_{i, j}-r_{i, j}\right),
\end{aligned}
$$

Where $\lambda=\left(\lambda_{i, j}: \forall i \in I, \forall j \in J\right), \sigma=\left(\sigma_{i, j}: \forall i \in I, \forall j \in J\right)$, and $\mu=\left(\mu_{i, j}: \forall i \in I, \forall j \in J\right)$ are vectors of Lagrange multipliers [15] corresponding to the bandwidth requirement constraint of (12) and the feasible region of $\mathrm{r}$ vector from (13) and (14) with $\sigma_{i, j}, \mu_{i, j} \geq 0$. The dual function can be expressed as

$$
h(\lambda, \sigma, \mu)=\max _{\mathbf{r}} L(\mathbf{r}, \lambda, \sigma, \mu)
$$

and the dual problem corresponding to the primal problem of (11) is

$$
\min _{(\sigma, \mu \geq 0), \lambda} h(\lambda, \sigma, \mu) \text {. }
$$

As the primal problem of $(11)\{(14)$ is a convex optimization problem, a strong duality exists. The optimal values for the primal and dual problems are equal. As a result, it is appropriate to solve the primal problem through its dual problem of (17). Hence, the optimum rate allocation $r_{i, j}$ for fixed value of dual variables $(\lambda, \sigma$ and $\mu$ ) can be calculated by applying the Karush-Kuhn-Tucker (KKT) conditions [15] on (17), and we have

$$
\frac{\partial U_{u s e r}(r, p)}{\partial r_{i, j}}+\lambda_{i, j}+\sigma_{i, j}-\mu_{i, j}=0 \text {. }
$$

Using the utility function of (2), we can conclude the optimal rate distribution $r_{i, j}$. Therefore, the optimal solution can be calculated as

$$
r_{i, j}=\frac{C_{i, j}\left(\kappa-p_{i, j}+\lambda_{i, j}+\sigma_{i, j}-\mu_{i, j}\right)}{2 \kappa} .
$$

Here, a gradient descent method can be applied to calculate the optimum values for dual variables $(\lambda, \sigma$ and $\mu)$ to solve (19), given by

$$
\begin{aligned}
\lambda_{i, j}(t+1) & =\left[\lambda_{i, j}(t)-\gamma_{1}\left(r^{\min }-\sum_{i \in I} \sum_{j \in J} r_{i, j}(t)\right)\right]^{+}, \\
\sigma_{i, j}(t+1) & =\left[\sigma_{i, j}(t)-\gamma_{2}\left(r_{i, j}(t)\right)\right]^{+}, \\
\mu_{i, j}(t+1) & =\left[\mu_{i, j}(t)-\gamma_{3}\left(C_{i, j}-r_{i, j}(t)\right]^{+},\right.
\end{aligned}
$$

where $t$ is the time index of iteration and $\gamma_{s}$ with $\mathrm{s}=\{1,2,3\}$ is a sufficiently small fixed step 
size. Convergence towards the optimum solution is guaranteed since the gradient of (19) satisfies the Lipschitz continuity condition [15]. As a result, the resource allocation $r_{i, j}$ converges to the optimum solution.

The proposed decomposition method [17] for the optimization problem of (11)-(14) is summarized as shown in Algorithm 1. In general, the complexity of the gradient descent method is $\mathrm{O}\left(1 / \gamma_{s}\right)$ [18]. Depending on the step size $\gamma_{s}$, the number of iterations can be decided.

Theorem 1 There exists at least one Nash equilibrium (NE) in the competition game.

Proof

Note that the strategy $p_{i, j}$ is a nonempty, convex, and compact subset of some Euclidean space $R^{N X N}$. Then, a utility function $U_{\text {ser }, i}(p)$ is a non-decreasing function in $r_{i, j}$. The resource allocation algorithm at the follower side represents a continuous function of resource distribution and it is quasi-convex in each $p_{i, j}$ from Proposition 1 . Therefore, by [18], the game has at least one Nash equilibrium.

Theorem 2 A Nash equilibrium exists in the non-cooperative pricing game (NPG) between service providers.

Proof

NE exists if the game satisfies the following properties:

- Strategy $p_{i, j}$ is a nonempty, convex, and compact subset of some Euclidean space $R^{N X N}$.

- A utility function $U_{\text {ser }, i}(p)$ is continuous in $\mathbf{p}$ and concave in $p_{i, j}$. Strategy space is defined to be positive and satisfies boundary condition in (16)-(17) with the other given other strategies. Therefore, it is a nonempty, convex, and compact subset of the Euclidean space $R^{N X N}$. Furthermore, according to proof in Proposition 1, an NE exists in game NPG.

Proposition 2 The resource allocation algorithm at the follower side (Algorithm 1) is quasi-convex in $p_{i, j}$.

Proof

Consider other prices $p_{-(i, j)}$ are fixed. Then when we increase the price from zero to $p_{i, j}$ gradually, resource allocation $r_{i, j}{ }^{*}$ will remain equal or decrease. After that, when we continue to increase $p_{i, j}$ until infinite value, $r_{i, j}{ }^{*}$ will reach to value $\max \left(0, r^{\min }-\sum_{(l, m) \neq(i, j)} C_{l, m}\right)$. Therefore, the resource allocation algorithm at the follower side is a non-increasing function in $p_{i, j}$. From [20], since a function that is non-increasing or non-decreasing everywhere is quasi-convex, we prove that the resource allocation algorithm at the follower side of Algorithm 1 is quasi-convex in $p_{i, j}$.

\subsection{Optimization Problem at the Leader-Level}

We can use the following gradient iteration algorithm to obtain the SE [13]. In Algorithm 2, $\sigma$ is the iterative step size of the price, $U_{s e r}=\left\{U_{s e r, 1}, U_{s e r, 2}, \ldots, U_{s e r, I}\right\}, \nabla U_{s e r}(p[t], r[t])$ is the gradient with $\partial U_{\text {ser }, i}(p[t], r[t]) / \partial p_{i, j}$. Similar to Algorithm 1, the complexity of the gradient descent method is $\mathrm{O}(1 / \sigma)$. 
Algorithm 1 A Gradient Rate Allocation Algorithm

1: Parameters: user utility $U_{\text {user }}(2)$, its available capacity $C_{i, j}$, price for bandwidth, and minimum bandwidth requirement $r^{\text {min }}$

2: Initialization: set $t=0$ and dual variables $(\lambda, \sigma$ and $\mu$ ) are equal to some non-negative values except for $\lambda$.

3: Repeat the iteration

(a) Calculating rate allocation vector by computing by computing (19).

(b) Updates dual variables with the gradient iterate (20)-(22).

(c) Until $\|r[t+1]-r[t]\| \leq \epsilon "$.

End iteration

Algorithm 2 Gradient Iteration Algorithm for Stackelberg Equilibrium

1: Initialization: randomly given a price $p_{i, j}$ over interface $j$ by service provider $i$

2: Repeat the iteration

(a). The user decides on which service providers to get the bandwidth from and how much bandwidth to get from Algorithm 1

(b). The service providers update the prices:

$p[t+1]=p[t]+\sigma \nabla \mathrm{U}_{\mathrm{ser}}(p[t], r[t])$.

(c). Until $\|p[t+1]-p[t]\| \leq \in$ ".

End iteration

\section{Theoretical Analysis of the Proposed Stackelberg Game}

\subsection{Theoretical Analysis of the Proposed Stackelberg Game}

$$
L(\mathbf{r}, \lambda)=U_{\text {user }}(\mathbf{r}, \mathbf{p})+\sum_{i \in I} \sum_{j \in J} \lambda_{i, j}\left(r_{i, j}-r^{\mathrm{min}}\right),
$$

where $\lambda$ is the Lagrange multiplier.

To solve the problem, the necessary and sufficient conditions for optimality are then given by the KKT conditions:

$$
\begin{array}{ll}
\frac{\partial U_{u s e r}\left(r^{*}, \mathbf{p}\right)}{\partial r_{i, j}}+\lambda_{i, j}^{*}=0, & \text { for } \forall i \in I, \forall j \in J, \\
\sum_{i \in I} \sum_{j \in J} r_{i, j}^{*}-r^{\min }=0 . &
\end{array}
$$

Therefore, from equation (13) and (14), for the given service requirement $r^{\min }$, the optimal rate allocation $r_{i, j}{ }^{*}$ at interface $j$ of service provider $i$ can be obtained as follows:

$$
\begin{aligned}
r_{i, j}^{*}= & \frac{C_{i, j}}{2 \kappa}\left(\frac{C_{i, j}}{X}-1\right) p_{i, j}+\frac{C_{i, j}}{2 \kappa} \frac{\sum_{(l, m) \neq(i, j)} C_{l, m} p_{l, m}}{X} \\
& +\frac{C_{i, j}}{X} r^{\text {min }},
\end{aligned}
$$

where $X=\sum_{i \in I} \sum_{j \in J} C_{i, j}$.

Here, to satisfy the feasible region of the optimal rate vector, we assume that a weight factor $\kappa$ should satisfy the below constraint, when the prices and other weight factors are given.

A) Positivity of $r^{*}\left(r^{*} \geq 0\right)$

The optimal rate over interface $i$ should be greater than or equal to 0 . 


$$
\kappa>\frac{1}{2 r^{\min }}\left\{\left(X-C_{i, j}\right) p_{i, j}-\sum_{(l, m) \neq(i, j)} C_{l, m} p_{l, m}\right\} .
$$

B) Capacity constraint of $r^{*}\left(r^{*}<C_{i, j}\right)$

The optimal rate allocation over interface $i$ of the service provider $j$ should be less than the available bandwidth $\left(C_{i, j}\right)$.

$$
\kappa>\frac{1}{2\left(X-r^{\min }\right)}\left\{\left(C_{i, j}-X\right) p_{i, j}+\sum_{(l, m) \neq(i, j)} C_{l, m} p_{l, m}\right\} .
$$

Here, because $\kappa$ is larger than 0 , we can conclude the $\kappa$ should satisfy below constraints

$$
\kappa>\max \{0,(15),(16)\} \text {. }
$$

Proposition 3 The optimal rate allocation $r_{i, j}{ }^{*}$ at the interface $j$ of the service provider $i$ decreases with its price $p_{i, j}$ increment, when other prices are fixed.

Proof

Taking the first-order derivative of $r_{i, j}{ }^{*}$, we have

$$
\frac{\partial r_{i, j}^{*}}{\partial p_{i, j}}=\frac{C_{i, j}}{2 \kappa}\left(\frac{C_{i, j}}{X}-1\right) .
$$

Since $\frac{C_{i, j}}{2 \kappa}$ and $\frac{C_{i, j}}{X}-1$ are the positive and negative values, respectively, $\frac{\partial r_{i, j}^{*}}{\partial p_{i, j}}$ is less than 0 . Therefore, $r_{i, j}{ }^{*}$ decreases with $p_{i, j}$.

\subsection{Maximizing the Service Provider Utility}

In this section, we analyze the leaders' strategies of the Stackelberg game. In this case, we assume that there are multiple leaders in the area where users can access ANs of service providers and compete with each other to maximize their own utilities by selecting their pricing strategies based on the consideration of the best response by the followers. Furthermore, according to Theorem 1-3, the existence of and the uniqueness of an NE is provided for the proposed service provider game. Finally, according to Theorems 4 and 5, we can conclude that there is a unique SE in the proposed two-level game between the service providers and the users.

\subsubsection{Analysis of the Service Provider Pricing Game}

Based on the analytical result of the follower rate allocation game, the leader of the Stackelberg game as service providers can optimize its strategy $p_{i}$ in order to maximize the utility defined at (5), which forms the NPG. Thus, from (9), the best response of leaders could be rewritten as :

$$
\mathbf{B}_{\mathbf{i}, \mathbf{j}}\left(p_{-\mathbf{i}, \mathbf{j}}\right)=\arg \max _{p_{i, j}} U_{s e r, i}\left(p_{i, j}, p_{-i, j}, r^{*}(\mathbf{p})\right)
$$

Where $p_{i, j}$ and $p_{-i, j}$ is the pricing strategy of the service provider $i$ for the AN $j$ and all other pricing strategy except $p_{i, j}$, respectively. In addition, $r^{*}(\mathbf{p})$ is the optimal rate allocation vector of followers with the given pricing $\mathbf{p}$ of leaders.

Substituting (4) and (15) into (19), taking the derivative of User to $p_{i, j}$, we have 


$$
\begin{aligned}
\frac{\partial U_{s e r, i}}{\partial p_{i, j}}=r_{i, j}^{*} & +p_{i, j} \frac{\partial r_{i, j}^{*}(\mathbf{p})}{\partial p_{i, j}}+\sum_{m \in \mathcal{J}, m \neq j} p_{i, m} \frac{\partial r_{i, m}^{*}(\mathbf{p})}{\partial p_{i, m}} \\
& -\sum_{m \in \mathcal{J}} c_{p} \frac{1-q_{i, m}}{C_{i, m}} \frac{\partial r_{i, m}^{*}(\mathbf{p})}{\partial p_{i, m}} P_{\max , i, m} .
\end{aligned}
$$

where $Y_{i, j}=\left(1-q_{i, j}\right) P_{\text {max,i,j }}$ Solving the above equations of $p_{i, j}$, we denote the solution of best response as $p_{i, j}$.

Definition 1 A pricing vector $\mathbf{p}=\left\{p_{i, j}\right\}_{I X J}$ is a NE of the NPG if, for every $i$ and $j, U_{\text {ser }, i}\left(p_{i, j}\right.$, $\left.p_{-i, j}\right) \geq U_{\text {ser }, i}\left(p_{i, j}, p_{-i, j}\right)$ for all $p_{i, j} \in \mathrm{p}$, where $U_{\text {ser }, i}\left(p_{i, j}, p_{-i, j}\right)$ is the resulting pricing strategy for the interface $j$ th of $i$ th service provider given the other interface pricing result $p_{-i, j}$.

Proposition 4 The utility function $U_{s e r, i}$ of service provider $i$ is concave in its own price $p_{i}$, when the other service provider prices are fixed and its optimal amount of rate is allocated from the user.

Proof

Taking the second-order derivative of $U_{\text {ser, }, \text { i, we have }}$

$$
\frac{\partial^{2} U_{s e r, i}}{\partial^{2} p_{i, j}}=\frac{C_{i, j}}{\kappa}\left(\frac{C_{i, j}}{X}-1\right) .
$$

Since $C_{i, j} / \kappa$ and $C_{i, j} / X-1$ are the positive and negative values, respectively, $\partial^{2} U_{s e r, i} / \partial^{2} p_{i, j}$ is less than 0 . Therefore, User;i is concave with respect to $p_{i, j}$.

From equations (19)-(22), we can conclude a close form of the best response function $B_{i, j}\left(p_{-i, j}\right)$ as below

$$
\begin{aligned}
B_{\mathbf{i}, \mathbf{j}}\left(p_{-i, \mathbf{j}}\right)= & \frac{c_{p} Y_{i, j}}{2 C_{i, j}}+\frac{\sum_{(l, m) \neq(i, j)} C_{l, m} p_{l, m}+2 \kappa r^{\min }}{2\left(X-C_{i, j}\right)} \\
& +\frac{\sum_{m \neq j}\left(C_{i, m} p_{i, m}-c_{p} Y_{i, m}\right)}{2\left(X-C_{i, j}\right)} .
\end{aligned}
$$

Next, we prove that the service provider level game has a unique NE. Therefore, according to Definition 1, the NE provides a set of prices such that none of the service providers can increase its individual utility by choosing a different price, given the prices offered by the other service providers. The key aspect of the uniqueness is proof to realize that the best response function in (24) is a standard function [21]. A function is said to be standard if it satisfies the following properties:

A) Positivity: $B_{\mathbf{i}, \mathbf{j}}\left(p_{-\mathbf{i}, \mathbf{j}}\right)>0$.

B) Monotonicity: $p_{-i, j} \geq p_{-i, j}^{\prime}, B_{\mathbf{i}, \mathbf{j}}\left(p_{-\mathbf{i}, \mathbf{j}}\right) \geq B_{\mathbf{i}, \mathbf{j}}\left(p_{-i \mathbf{i},}^{\prime}\right)$.

C) Scalability: $\mu B_{\mathrm{i}, \mathbf{j}}\left(p_{-\mathrm{i}, \mathbf{j}}\right)>B_{\mathrm{i}, \mathrm{j}}\left(\mu p_{-\mathbf{i}, \mathbf{j}}\right), \forall \mu>1$.

Theorem 3 The best response function $B_{i, j}\left(p_{-i, j}\right)$ of an arbitrary service provider $i$ is standard. Therefore, a unique NE exists in the NPG between service providers.

Proof

A) Positivity

The first term and second term of the best response function (24) are always greater than 0 . In 
addition, the service providers do not set the price at each of the ANs that returns the decit in (5), and hence, we can assume that the below inequality always holds for any $i$ and $j$.

$$
\begin{array}{r}
p_{i, j} r_{i, j}^{*}-c_{p} Y_{i, j} / C_{i, j} r_{i, j}^{*}-c_{p} q_{i, j} \cdot P_{\text {max }, i, j}>0, \\
\text { for } \forall i \in I, \forall j \in J, \\
=>C_{i, j} p_{i, j}-c_{p} Y_{i, j}>C_{i, j} c_{p} q_{i, j} \cdot P_{\text {max }, i, j} / r_{i, j}^{*}, \\
\text { for } \forall i \in I, \forall j \in J .
\end{array}
$$

From (26), we can conclude $C_{i, m} p_{i, m}-C_{p} Y_{i, m}$ is always a positive value for any $i$ and $j$, so the third term in (24) is also positive. Thus, the best response function (24) is always positive.

B) Monotonicity

Here, the problem can be reduced to provide $\partial B_{s e r, i}\left(p_{-i, j}\right) / \partial p_{-i, j} \geq 0$. Taking the derivative of the best response function $B_{i, j}\left(p_{-i, j}\right)$ to $p_{i, k}$, we obtain

C) Scalability

$$
\frac{\partial B_{i, j}\left(p_{-i, j}\right)}{\partial p_{i, k}}=\frac{C_{i, k}}{X-C_{i, j}}>0 .
$$

Based on (24), we can obtain

$$
\begin{aligned}
\mu B_{\mathbf{i}, \mathbf{j}}\left(p_{-\mathbf{i}, \mathbf{j}}\right)= & \mu\left\{\frac{C_{p} Y_{i, j}}{2 C_{i, j}}+\frac{\sum_{(l, m) \neq(i, j)} C_{l, m} p_{l, m}+2 \kappa r^{\min }}{2\left(X-C_{i, j}\right)}\right. \\
+ & \left.\frac{\sum_{m \neq j}\left(C_{i, m} p_{i, m}-C_{p} Y_{i, m}\right)}{2\left(X-C_{i, j}\right)}\right\} . \\
B_{\mathbf{i}, \mathbf{j}}\left(\mu p_{-\mathbf{i}, \mathbf{j}}\right)= & \frac{c_{p} Y_{i, j}}{2 C_{i, j}}+\frac{(l, m) \neq(i, j)}{2\left(X-C_{l, m} p_{l, m}+2 \kappa r^{\min }\right.} \\
& +\frac{\sum_{m \neq j}\left(\mu C_{i, m} p_{i, m}-C_{p} Y_{i, m}\right)}{2\left(X-C_{i, j}\right)} .
\end{aligned}
$$

Based on (28) and (29), we can obtain

$$
\begin{gathered}
\mu B_{\mathbf{i}}\left(p_{-\mathbf{i}}\right)-B_{\mathbf{i}}\left(\mu p_{-\mathbf{i}}\right)=(\mu-1) \frac{c_{p} Y_{i, j}}{2 C_{i, j}} \\
+(\mu-1) \frac{2 \kappa r^{\min }-c_{p} \sum_{m \neq j} Y_{i, m}}{2\left(X-C_{i, j}\right)} .
\end{gathered}
$$

Therefore, for all $\mu>1$, scalability is satisfied with the given electricity price $c_{p}$ from the retailer having an upper bound as below

$$
c_{p}<\frac{2 \kappa r^{\min }}{\sum_{m \neq j} Y_{i, m}-Y_{i, j} \frac{X-C_{i, j}}{C_{i, j}} .}
$$




\subsubsection{Existence and Uniqueness of the Stackelberg Equilibrium for the Proposed Two-Level Game}

For a Stackelberg game, existence and uniqueness of an SE are two desirable properties. In this subsection, we will prove that solutions $r_{i, j}^{*}(\mathbf{p})$ and $p^{*}{ }_{i, j}(i \in \mathrm{I}, j \in \mathrm{J})$ are the SE for the proposed game.

Theorem 4 An SE exists in the proposed two-level game.

Proof

To prove the existence of SE, we prove that the solution $r_{i, j}^{*}(\mathbf{p})$ in (15) is the global optimum that maximizes the user utility function $\left(U_{\text {user }}\right)$. Therefore, we verify that $r_{i, j}^{*}(\mathbf{p})$ in (15) meets the KKT conditions. Based on (11), we get

$$
\nabla^{2} L\left(r_{i, j}^{*}(\mathbf{p})\right)=-\frac{2 \kappa}{C_{i, j}}<0
$$

Therefore, $r_{i, j}^{*}(\mathbf{p})$ in (14) is the global optimum that maximizes the user utility function $\left(U_{\text {user }}\right)$. $r_{i, j}^{*}(\mathbf{p})$ satisfies (6) and is the SE $\left(r^{S E}\right)$ in (9). Because of the concavity of $U_{u s e r, i}$, the service provider $i$ can always find its optimal price $p_{\mathrm{i}, \mathrm{j}}^{*}$.
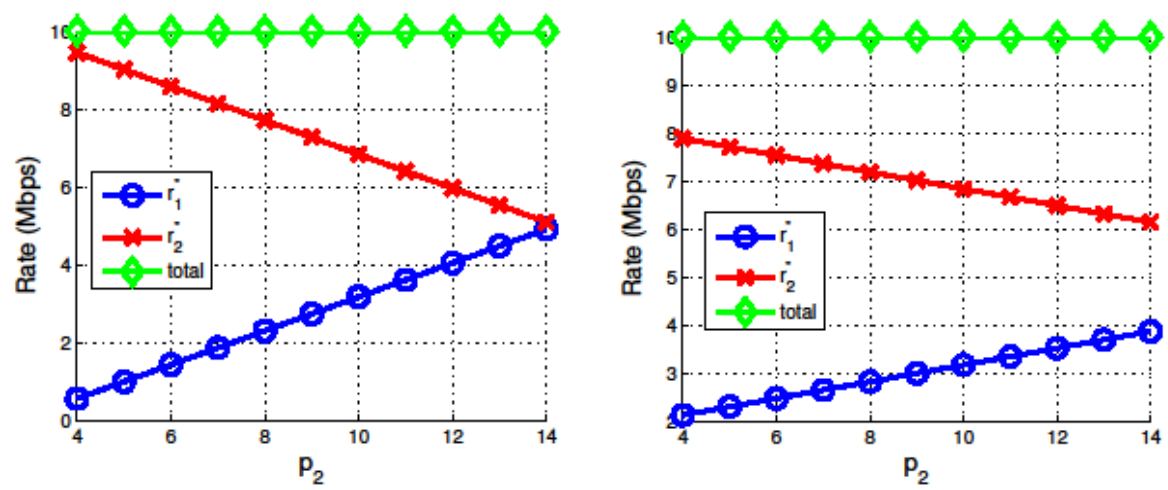

Fig. 2. Rate allocation to AN1 and AN2 (a) $\kappa=4$ and (b) $\kappa=10$ as the price offered by service provider 2 varies

Theorem 5 A unique SE exists in the proposed Two Level game.

Proof

Since the best response function $B_{i, j}\left(p_{-i, j}\right)$ of an arbitrary service provider $i$ is standard from Theorem 2, the service provider pricing game has the unique NE. Therefore, the proposed game has the unique SE.

\section{Numerical Results}

The rate allocation behavior of the users dependent on various price profiles needs to be analzyed. For this, we assume there are different types of ANs. For convenience, we name the ANs as AN1 and AN2, which operate at different channel bandwidths and have maximum capacities of $5.1 \mathrm{Mbps}$ and $11 \mathrm{Mbps}$, respectively, such that they do not interfere with each other. Based on the linear relationship between the transmission and the operational power consumptions, we consider that the maximum operational power is $38 \mathrm{~W}$ and $50 \mathrm{~W}$ for AN1 
and AN2, receptively [16]. In addition, the portion of the fixed power consumption to $P_{\max , i}\left(q_{i}\right)$ is $40 \%$ and $50 \%$ for AN1 and AN2, respectively. Then, a weight factor for the satisfaction from the bandwidth $(\xi)$ is set by 50 .

\subsection{Rate Allocation according to Service Provider Price}

We check how the rate allocation to AN1 and AN2 is a affected by the service price offered by the service provider 2 in the proposed game. Fig. 2 shows that the rate allocation to AN2 linearly decreases with the price $p_{2}$ while $p_{1}$ is fixed in Proposition 5.1. On the other hand, the amount of rate allocation to AN1 correspondingly increases for guaranteeing the $r^{\mathrm{min}}$. This intuitive result about rate allocation versus price is based on Proposition 2.1. Furthermore, in
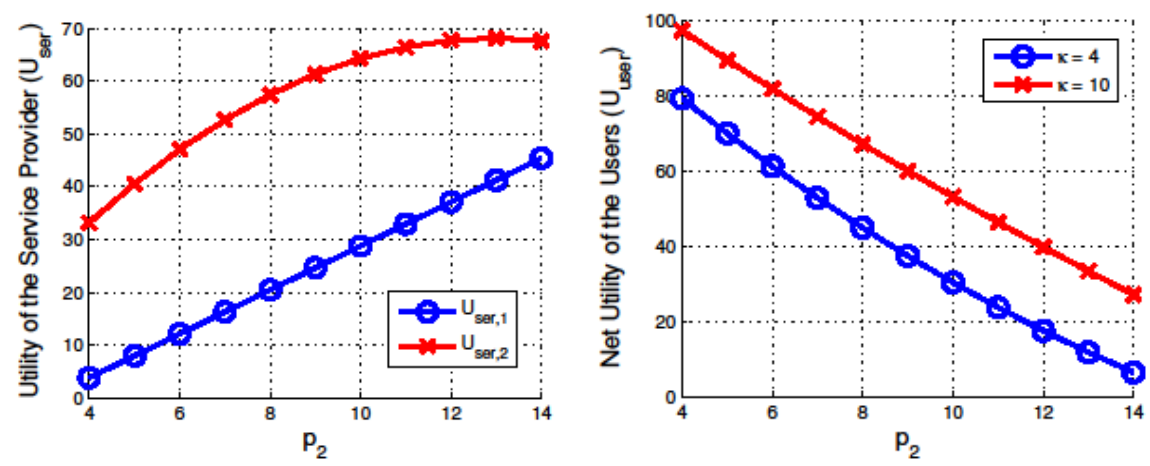

Fig. 3. Utility of (a) service providers and (b) users $(\kappa=4,10)$ as the price offered by service provider 2 varies

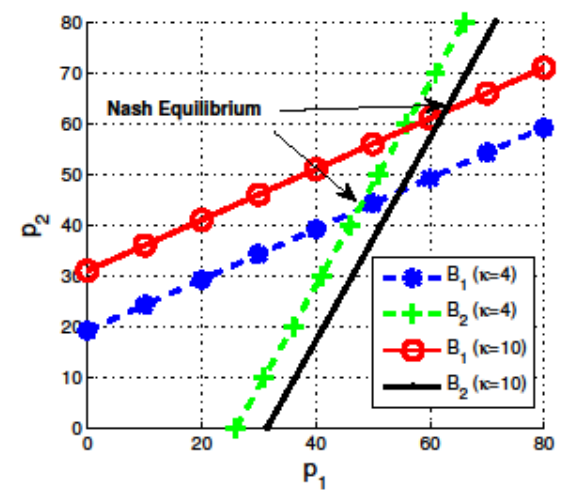

Fig. 4. Existence and uniqueness of the NE of the pricing game among service providers
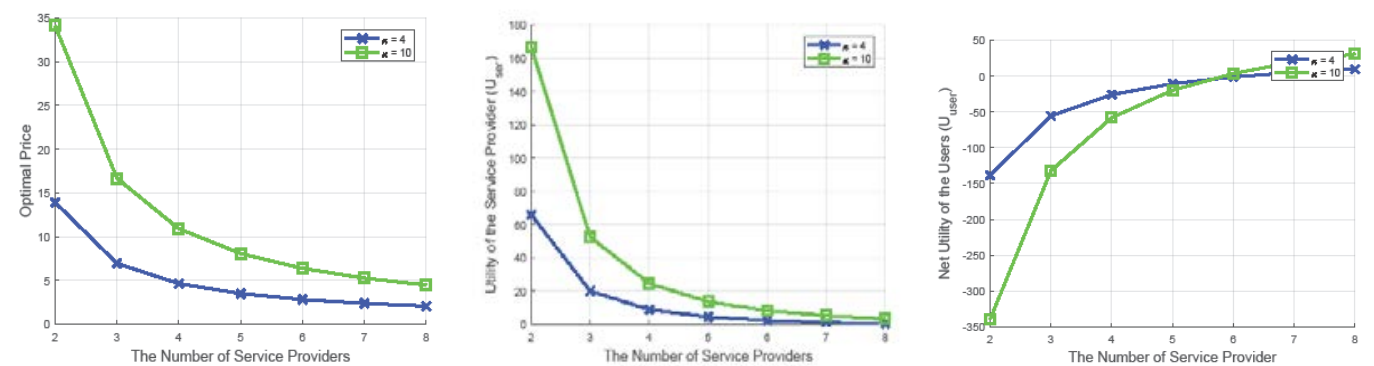

Fig. 5. (a) Optimal price and the utility of (b) users and (c) service providers at the SE as the number of service providers varies 
Fig. 2(b), as compared to Fig. 2(a), as the value of $\kappa$-which is the weight factor of delay-increases, the rate allocation to AN1 decreases to a value lesser than that in Fig. 2(a). This is because the increased value of $\kappa$ gives more penalties to the congestion.

\subsection{Utility of Service Providers and Users according to the Service Provider Price}

Based on the result of Fig. 2, we analyze the utility of the service provider and users as the price offered by the service provider 2 varies. Fig. 3(a) shows that the utility function of the service provider 2 is concave in price $p_{2}$ from Proposition 4.1, and the utility of the service provider 1 increases with the price $p_{2}$. For each price offered by the service provider 2, the service provider 1 offers a price based on the best response strategy for maximizing its own utility.

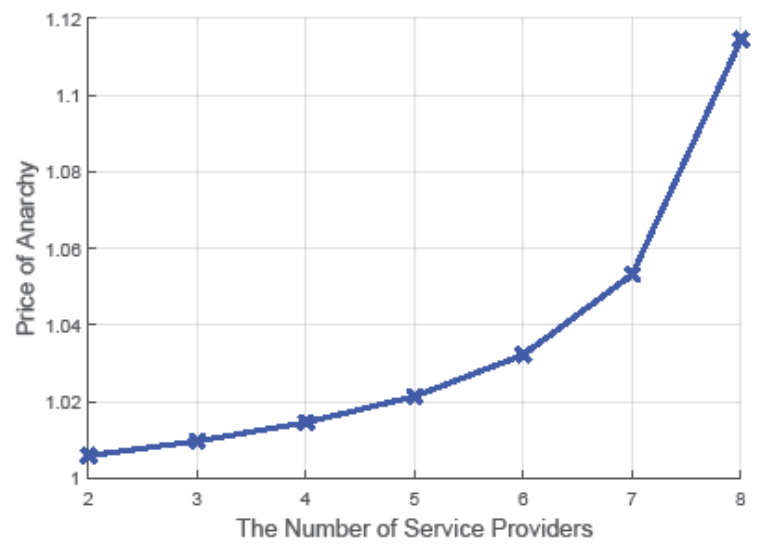

Fig. 6. Price of anarchy as the number of service providers varies

The amount of rate allocation to AN2 decreases with the increase of the price offered by the service provider 2. On the other hand, the rate allocation to AN1 correspondingly increases. In addition, the increased price of the service provider 2 affects the best strategy of service provider 1 . Thus, the utility of the users decrease with price $p_{2}$ as shown in Fig. 3(b). In addition, as the value of $\kappa$ increases, the net utility of users gives more weight to the impact of delay. Hence, a higher net utility of users is achieved owing to the lesser impact of the price. Fig. 4 shows the best response functions and the NE of the competitive price of two service providers. This gure graphically shows the existence and uniqueness of the NE. As shown this, there is only one point of intersection, which is the NE of the prices offered to the users by the service providers. In this case, as the value of $\kappa$ increases, the NE moves into a higher value owing to the lesser impact on the price.

\subsection{Utility of Service Providers and Users according to the Number of Service Providers}

We investigate how the number of service providers (leaders) affects the utility of users and service providers as well as the optimal price. Here, we assume that the specification of different service provider ANs is identical to that of AN1. As shown Fig. 5(a), the optimal price decreases with the increase in the number of service providers. As the number of service providers increases, the competition among them becomes more severe, and therefore, the average price decreases. Thus, the utility of the service provider decreases owing to the 
reduced optimal price shown in Fig. 5(b). On the other hand, Fig. 5(c) shows that the net utility of the users increases with the increase of the number of service providers.

Moreover, with an increase in the value of $\kappa$ (from $\kappa=4$ to $\kappa=10$ ), the optimal price of the service providers increases because the price affects the net utility of the users lesser than the delay penalty shown in Fig. 5(a). Therefore, the utility of the service provider also increases shown in Fig. 5(b). In Fig. 5(a), when the number of the service providers is low and $\kappa$ is 10 , the net utility of the users has a lower value owing to the high price from the service provider and the impact of the delay penalty. However, as the number of service providers increases to more than five, because of the reduced price from severe competition among the service providers and achieving less delay penalty over various AN options, the net utility of users crosses each other. In Fig. 6, we demonstrate the efficiency of the equilibrium by illustrating the price of anarchy with respect to the number of service providers. The result confirms that the equilibrium solution of the non-cooperative game has small performance loss in terms of social welfare, which is always lower bounded by half of the optimal centralized solution of the cooperative game.

\section{Conclusion}

This paper investigated multi-homing for rich multimedia applications in heterogeneous wireless ANs. We propose a joint pricing and load distribution mechanism that guarantees users the minimum QoS, improves the service provider revenue, and reduces their operational expenditure related to power consumption. The proposed mechanism is based on a multileader-, multifollower Stackelberg game among service providers and users. Users can optimally conduct resource allocation in detail to maximize their utility related to delay penalty and price of the resource allocation. Besides, service providers can maximize their revenue in response to the user reaction. The results demonstrate that our proposed approach is guaranteed to have a unique equilibrium solution that can maximize the payoff for all participating mobile users and service providers.

\section{References}

[1] T. Braud, F. H. Bijarbooneh, D. Chatzopoulos, and P. Hui, "Future networking challenges: The case of mobile augmented reality,” in Proc. of IEEE ICDCS '17, Atlanta, GA, USA, June 2017. Article (CrossRef Link)

[2] F. Teka, C. H. Lung, and S. A. Ajila, "Nearby live virtual machine migration using cloudlets and multipath TCP,” Journal of Cloud Computing, vol. 5, no. 1, 12, 2016. Article (CrossRef Link)

[3] S. Hyrynsalmi, A. Suominen, and M. Mäntymäki, "The inuence of developer multi-homing on competition between software ecosystems,” Journal of Systems and Software, vol. 111, pp. 119 127, 2016. Article (CrossRef Link)

[4] S. Prabhavat, H. Nishiyama, N. Ansari, and N. Kato, "On load distribution over multipath networks,” IEEE Communications Surveys Tutorials, vol. 14, no. 3, 662-680, Third 2012.

Article (CrossRef Link)

[5] T. V. Seenivasan and M. Claypool, "Cstream: neighborhood bandwidth aggregation for better video streaming,” Multimedia Tools and Applications, vol. 70, no. 1, 379-408, 2014. Article (CrossRef Link)

[6] M. P. Guimarães, D. R. C. Dias, J. H. Mota, B. B. Gnecco, V. H. S. Durelli, and L. C. Trevelin, "Immersive and interactive virtual reality applications based on 3D web browsers," Multimedia Tools and Applications, pp. 1-15, 2016. Article (CrossRef Link) 
[7] A. Petlund, P. Beskow, J. Pedersen, E. S. Paaby, C. Griwodz, and P. Halvorsen, "Improving SCTP retransmission delays for timedependent thin streams,” Multimedia Tools and Applications, vol. 45, no. 1, pp.33-60, 2009. Article (CrossRef Link)

[8] Y. Cui, T. Li, C. Liu, X. Wang, and M. Khlewind, "Innovating transport with QUIC: Design approaches and research challenges,” IEEE Internet Computing, vol. 21, no. 2, 72-76, Mar 2017. Article (CrossRef Link)

[9] C. López, R. Agüuero, J. Choque, and L. Muñoz, "On the equilibrium of resource allocation for heterogeneous wireless access networks," in Proc. of IEEE PIMRC'12, pp. 1049-1054, Sept 2012. Article (CrossRef Link)

[10] T. Dutta, S. Sailendra, and P. Balamuralidhar, "An efficient bandwidth aggregation algorithm using game theory for multimedia transmission," in Proc. of IEEE PIMRC'14, pp. 1783-1787, Sept 2014. Article (CrossRef Link)

[11] M. Ismail, K. Qaraqe, and E. Serpedin, "Cooperation incentives and downlink radio resource allocation for green communications in a heterogeneous wireless environment," IEEE Transactions on Vehicular Technology, vol. 65, no. 3, pp. 1627-1638, March 2016.

Article (CrossRef Link)

[12] S. Yun, J. Lee, S. H. S. Newaz, and J. K. Choi, "Energy efficient pricing scheme for multi-homing in heterogeneous wireless access networks: A game theoretic model and its analysis," in Proc. of IEEE WCNC'15, pp. 1672-1677, March 2015. Article (CrossRef Link)

[13] D. Niyato, E. Hossain, and Z. Han, "Dynamics of multiple-seller and multiple-buyer spectrum trading in cognitive radio networks: A game-theoretic modeling approach," IEEE Transactions on Mobile Computing, vol. 8, no. 8, pp. 1009-1022, Aug. 2009. Article (CrossRef Link)

[14] T. Alpcan and T. Basar, "A globally stable adaptive congestion control scheme for internet-style networks with delay,” IEEE/ACM Transactions on Networking, vol. 13, no. 6, pp. 1261-1274, Dec. 2005. Article (CrossRef Link)

[15] S. Boyd and L. Vandenberghe, "Convex Optimization," Cambridge University Press, Cambridge, 2004.

[16] K. Son, H. Kim, Y. Yi, and B. Krishnamachari, "Base station operation and user association mechanisms for energy-delay tradeoffs in green cellular networks," IEEE Journal on Selected Areas in Communications, vol. 29, no. 8, pp. 1525-1536, Sep. 2011. Article (CrossRef Link)

[17] D. P. Palomar and M. Chiang, "A tutorial on decomposition methods for network utility maximization," IEEE Journal on Selected Areas in Communications, vol. 24, no. 8, pp. 1439-1451, Aug. 2006. Article (CrossRef Link)

[18] G. Gordon and R. Tibshirani, "Lecture 5: Gradient desent revisited," Carnegie Mellon Univ., Fall 2012. [Online].

[19] A. Mas-Colell, M. D. Whinston, and J. R. Green, "Microeconemic Theory,” Oxford Univ. Press, Oxford, UK, 1995.

[20] J. A. dos Santos Gromicho, "Quasiconvex Optimization and Location Theory,” Springer, Berlin, Germany, 1998. Article (CrossRef Link)

[21] J. Zhang and Q. Zhang, "Stackelberg game for utility-based cooperative cognitive radio networks," in Proc. of ACM MobiHoc '09, pages 23-32, New Orleans, USA, May 2009. Article (CrossRef Link) 


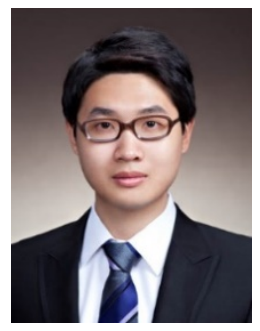

Joohyung Lee (S’09-M'14) is currently an Assistant Professor in the Department of Software, at Gachon University, South Korea. He received his B.S, M.S. and Ph.D. Degrees from the Korea Advanced Institute of Science and Technology (KAIST), Daejeon, South Korea, in 2008, 2010 and 2014, respectively. From 2012 to 2013, he was a Visiting Researcher with the Information Engineering Group, Department of Electronic Engineering, City University of Hong Kong, Hong Kong. From 2014 to 2017, he worked at Samsung Electronics as a Senior Engineer. He has contributed several articles to the International Telecommunication Union Telecommunication (ITU-T) and 3rd Generation Partnership Project (3GPP). His current research interests include resource allocation and optimization, with a focus on resource management for Future Media (e.g., Augmented Reality, Virtual Reality), 5G networks, green networks, cloud computing, smart grids (future power grids), and network economics.

Dr. Lee was an active member of the GreenTouch Consortium. He has been a Technical Reviewer for several conferences and journals, such as the IEEE COMMUNICATIONS LETTERS, the IEEE TRANSACTIONS ON VEHICULAR TECHNOLOGY, and Elsevier Computer Communications. He received a Best Paper Award at the Integrated Communications, Navigation, and Surveillance Conference in 2011. 